\title{
Synchronization of mouse islets of Langerhans by
}

glucose waveforms

Xinyu Zhang, Arij Daou, Tuan M. Truong, Richard Bertram and Michael G.

Roper

Am J Physiol Endocrinol Metab 301:E742-E747, 2011. First published 19 July 2011;

doi:10.1152/ajpendo.00248.2011

You might find this additional info useful...

This article cites 26 articles, 17 of which can be accessed free at:

http://ajpendo.physiology.org/content/301/4/E742.full.html\#ref-list-1

Updated information and services including high resolution figures, can be found at:

http://ajpendo.physiology.org/content/301/4/E742.full.html

Additional material and information about AJP - Endocrinology and Metabolism can be found at: http://www.the-aps.org/publications/ajpendo

This infomation is current as of September 27, 2011.

AJP - Endocrinology and Metabolism publishes results of original studies about endocrine and metabolic systems on any level of organization. It is published 12 times a year (monthly) by the American Physiological Society, 9650 Rockville Pike, Bethesda MD 20814-3991. Copyright (C) 2011 by the American Physiological Society. ISSN: 0193-1849, ESSN: 1522-1555. Visit our website at http://www.the-aps.org/. 


\title{
Synchronization of mouse islets of Langerhans by glucose waveforms
}

\author{
Xinyu Zhang, ${ }^{1}$ Arij Daou, ${ }^{2}$ Tuan M. Truong, ${ }^{1}$ Richard Bertram, ${ }^{2,3,4}$ and Michael G. Roper ${ }^{1,4}$ \\ ${ }^{1}$ Department of Chemistry and Biochemistry and ${ }^{2}$ Department of Mathematics and Programs in ${ }^{3}$ Neuroscience and \\ ${ }^{4}$ Molecular Biophysics, Florida State University, Tallahassee, Florida
}

Submitted 18 May 2011; accepted in final form 12 July 2011

Zhang X, Daou A, Truong TM, Bertram R, Roper MG. Synchronization of mouse islets of Langerhans by glucose waveforms. Am J Physiol Endocrinol Metab 301: E742-E747, 2011. First published July 19, 2011; doi:10.1152/ajpendo.00248.2011.-Pancreatic islets secrete insulin in a pulsatile manner, and the individual islets are synchronized, producing in vivo oscillations. In this report, the ability of imposed glucose waveforms to synchronize a population of islets was investigated. A microfluidic system was used to deliver glucose waveforms to $\sim 20$ islets while fura 2 fluorescence was imaged. All islets were entrained to a sinusoidal waveform of glucose $(11 \mathrm{mM}$ median, $1 \mathrm{mM}$ amplitude, and a 5-min period), producing synchronized oscillations of fura 2 fluorescence. During perfusion with constant $11 \mathrm{mM}$ glucose, oscillations of fura 2 fluorescence were observed in individual islets, but the average signal was nonoscillatory. Spectral analysis and a synchronization index $(\lambda)$ were used to measure the period of fura 2 fluorescence oscillations and evaluate synchronization of islets, respectively. During perfusion with glucose waveforms, spectral analysis revealed a dominant frequency at $5 \mathrm{~min}$, and $\lambda$, which can range from 0 (unsynchronized) to 1 (perfect synchronization), was $0.78 \pm 0.15$. In contrast, during perfusion with constant $11 \mathrm{mM}$ glucose, the main peak in the spectral analysis corresponded to a period of $5 \mathrm{~min}$ but was substantially smaller than during perfusion with oscillatory glucose, and the average $\lambda$ was $0.52 \pm 0.09$, significantly lower than during perfusion with sinusoidal glucose. These results indicated that an oscillatory glucose level synchronized the activity of a heterogeneous islet population, serving as preliminary evidence that islets could be synchronized in vivo through oscillatory glucose levels produced by a liver-pancreas feedback loop.

microfluidic; entrainment; interislet; calcium oscillations

PULSATILE IN VIVO INSULIN LEVELS with a period of 5-10 min are observed in normal individuals $(7,14)$. Compared with a constant insulin level, pulsatile insulin is more effective at inhibiting hepatic glucose production and inducing glucose uptake by peripheral tissues in vivo $(2,6,10,15)$. In type 2 diabetics, impaired plasma insulin pulses are observed, resulting in inefficient glucose clearance and exacerbating the problem of hyperglycemia $(7,14,17)$. Therefore, it is critical to understand the origin and nature of these in vivo insulin oscillations to understand how they become impaired in type 2 diabetics.

The conventional hypothesis of glucose-stimulated insulin secretion begins with glucose delivered across the cell membrane through specific glucose transporters (11). After the rate-limiting phosphorylation by glucokinase, oxidative metabolism leads to increases in the ratio of ATP to ADP. This increase in the ATP/ADP ratio closes ATP-sensitive $\mathrm{K}^{+}$channels, depolarizing the cell membrane, leading to the opening of

\footnotetext{
Address for reprint requests and other correspondence: M. G. Roper, Dept. of Chemistry and Biochemistry, Florida State University, 95 Chieftain Way, Dittmer Bldg., Tallahassee, FL 32306 (e-mail: roper@chem.fsu.edu).
}

voltage-dependent $\mathrm{Ca}^{2+}$ channels and an increase in the intracellular $\left[\mathrm{Ca}^{2+}\right]\left(\left[\mathrm{Ca}^{2+}\right]_{i}\right)$. The increase in $\left[\mathrm{Ca}^{2+}\right]_{\mathrm{i}}$ leads to insulin exocytosis via $\mathrm{Ca}^{2+}$-dependent processes. Exposure to a stimulatory glucose level results in pulsatile $\left[\mathrm{Ca}^{2+}\right]_{i}$ within a single islet of Langerhans, leading to pulsatile insulin secretion from the islet $(5,8)$. A potential mechanism for these oscillations is described by the Dual Oscillator Model, which postulates that oscillations in glycolysis produce the slow $(\sim 5 \mathrm{~min})$ oscillations in islet activity whereas feedback of $\mathrm{Ca}^{2+}$ onto ion channels produces faster oscillations (1). There has been much research into the mechanisms underlying single islet behavior, but much less is known about how the activity of the many islets within the pancreas is synchronized to yield a coherent pulsatile insulin level in the peripheral blood.

The observation of in vivo insulin pulses in humans implies that insulin secretion, and therefore the $\left[\mathrm{Ca}^{2+}\right]_{\mathrm{i}}$, of the $\sim 10^{6}$ islets within a pancreas is synchronized. There are two main hypotheses to explain this interislet synchronization: periodic stimulation of acetylcholine from intrapancreatic ganglia that entrains islets $(4,21,26)$ or a pancreas-liver feedback loop that produces oscillatory blood glucose levels, which then acts as a global metabolic signal to synchronize islets $(3,7,16)$. There may be redundancy between these mechanisms in vivo, and several pathways may be utilized to synchronize insulin output. However, there have been few in vitro experiments demonstrating synchronization of islets via any of these pathways.

To verify the possibility that glucose waveforms can synchronize islets, insulin release from single islets $(3,24)$, perfused pancreas $(3,24)$, and in vivo insulin levels $(9,18)$ have been entrained to pulsatile glucose levels. Other reports have not been able to demonstrate entrainment of 5-min insulin secretory pulses to a 10-min periodic glucose infusion (19) or $\left[\mathrm{Ca}^{2+}\right]_{\mathrm{i}}$ oscillations between cell clusters (25) to small-amplitude glucose pulses. The differences in the results may be due to the glucose profile applied (10-min glucose waveforms vs. 5-min waveforms) or islet preparation (whole islets vs. cell clusters). We have developed microfluidic devices that produce glucose profiles mimicking those found in vivo to explore islet synchronization $(27,28)$. We have found that sinusoidal glucose waveforms can entrain $\left[\mathrm{Ca}^{2+}\right]_{\mathrm{i}}$ within single islets, even to complex gradient profiles that included phase shifts in the glucose waves (27).

In this work, we build upon our previous report by demonstrating that multiple islets can be entrained to a glucose waveform, which synchronizes the islets. A microfluidic device was used to generate sinusoidal glucose waveforms with a 5-min period that were delivered to a chamber containing $\sim 20$ islets. This period of glucose wave was used since it is approximately the period of insulin oscillations observed in portal and peripheral blood of rodents and humans $(7,14)$. Changes in $\left[\mathrm{Ca}^{2+}\right]_{i}$ from all islets were imaged via fura 2 fluorescence as a marker of islet response. The average fura 2 
fluorescence from the islet population was quantified by a synchronization index $(\lambda)(20,25)$, which indicated that the degree of islet synchronization greatly increased upon initiation of the sinusoidal glucose waveform. These results provide preliminary evidence that pancreas-liver interactions may play a significant role in interislet synchronization in vivo.

\section{MATERIALS AND METHODS}

Chemicals and reagents. $\mathrm{HNO}_{3}, \mathrm{CaCl}_{2}, \mathrm{NaOH}$, and $\mathrm{NaCl}$ were purchased from EMD Chemicals, (Gibbstown, NJ). Polydimethylsiloxane was from Rogers (Carol Stream, IL). Glucose (dextrose), $\mathrm{MgCl}_{2}$, hydrofluoric acid, and Cosmic Calf Serum were from Fisher Scientific (Pittsburgh, PA). KCl, fluorescein, tricine, dimethyl sulfoxide (DMSO), and type XI collagenase were from Sigma (St. Louis, MO). Fura 2 acetoxymethyl ester (fura 2-AM), Pluronic F-127, RPMI 1640 with $11 \mathrm{mM}$ glucose, and penicillin-streptomycin were from Invitrogen (Carlsbad, CA). All solutions were made with Milli-Q (Millipore, Bedford, MA) $18 \mathrm{M} \Omega / \mathrm{cm}$ deionized water.

All buffer solutions used in the experiments were composed of 2.4 $\mathrm{mM} \mathrm{CaCl}_{2}, 125 \mathrm{mM} \mathrm{NaCl}, 1.2 \mathrm{mM} \mathrm{MgCl}_{2}, 5.9 \mathrm{mM} \mathrm{KCl}$, and $25 \mathrm{mM}$ tricine and different glucose solutions, as described below.

Microfluidic device. The device was fabricated as described previously (28). The microfluidic chip had two pneumatic pumps that delivered 13 and $3 \mathrm{mM}$ glucose into a $3.3-\mathrm{cm}-l o n g$ mixing channel $(220 \times 90 \mu \mathrm{m}$, width $\times$ depth). At the end of this channel, the mixing channel split into equal-length segments twice, producing four channels that delivered the glucose waves to the islet chamber. The chamber was drilled with a 0.035-in. diamond-tipped drill bit (Norton Abrasives, North Tonawanda, NY), which produced a 1-mm-diameter chamber. In all experiments, a thermofoil and thermocouple sensor (Omega Engineering, Stamford, CT) were attached to the microfluidic chip to maintain the temperature of the mixing channel and islet chamber at $37^{\circ} \mathrm{C}$. The pneumatic pumps were actuated via computercontrolled solenoid valves controlled by a data acquisition card (NI PCI-6221) and a program written in LabView (National Instruments, Austin, TX).

Isolation of islets of Langerhans. Islets of Langerhans were collected as described previously (27). For $\left[\mathrm{Ca}^{2+}\right]_{\mathrm{i}}$ monitoring, $1.0 \mu \mathrm{l}$ of $5.0 \mathrm{mM}$ fura 2-AM in DMSO and $1.0 \mu \mathrm{l}$ Pluronic F-127 in DMSO were mixed and transferred into $2 \mathrm{ml}$ of RPMI to form a final fura 2-AM concentration of $2.5 \mu \mathrm{M}$. Each batch of islets was incubated in this solution at $37^{\circ} \mathrm{C}$ and $5 \% \mathrm{CO}_{2}$ for $45 \mathrm{~min}$.

After incubation, islets were delivered slowly by gravity from a pipette into the islet chamber, where they settled onto the chamber bottom in a single layer. Since it has been reported that islets from particular mice have imprinted $\left[\mathrm{Ca}^{2+}\right]_{i}$ oscillation frequencies $(12$, 13 ), experiments were performed with islets from either a single mouse or multiple mice, as specified. Islets were perfused within the device with $3 \mathrm{mM}$ glucose for $5 \mathrm{~min}$ prior to recording fura 2 fluorescence.

Detection. The microfluidic device was placed on the stage of a Nikon Eclipse Ti microscope for all experiments. A broadband lamp (Lambda XL; Sutter Instruments, Novato, CA) was integrated with a filter wheel containing two filters (XF1093 and XF1094; Omega Optical, Brattleboro, VT) for excitation of fura 2 fluorescence. To minimize photobleaching of fura 2 fluorescence a shutter (Sutter Instruments) was used, which opened $150 \mathrm{~ms}$ for each filter every 10 s. The excitation light was focused onto the islet chamber through a $\times 10,0.5$ NA objective via a dichroic mirror (XF2002). The fura 2 emission was collected by the same objective, passed through the dichroic mirror and an emission filter (XF3043), and imaged by a charge-coupled device camera (Cascade; Photometrics, Tucson, AZ). NIS Elements (Nikon, Melville, NY) was used to control the Sutter filter wheel, shutter, and charge-coupled device camera. All measurements of fura 2 fluorescence are reported as the ratio of background- corrected fura 2 emission at $520 \mathrm{~nm}$ upon excitation by 340 and 380 $\mathrm{nm}\left(\mathrm{F}_{340} / \mathrm{F}_{380}\right)$.

Spectral analysis. Spectral analysis using a fast Fourier transform (FFT) was performed on the average fura 2 fluorescence signal obtained from integrating the $\mathrm{F}_{340} / \mathrm{F}_{380}$ ratio from all islets. For all experiments the data from 30 to $40 \mathrm{~min}$ were used for the spectral analysis, i.e., the last $10 \mathrm{~min}$ of the fura 2 fluorescence traces. Each of these experiments was repeated four times.

Synchronization index $(\lambda)$. $\lambda$ is a measurement of the phase difference between two oscillators and is calculated when the phase of the first oscillator attains a fixed value. Computer code for calculating $\lambda$ of two oscillators was provided elsewhere (25) but was adapted in this report for the analysis of more than two oscillators. For example, in a population of 20 islets, each islet was numbered from 1 to 20 . The extent of synchronization was then determined between islets 1 and 2, islets 1 and 3, etc., until islet 1 was compared with the 19 other islets. This process was then repeated, now comparing islet 2 with all other islets, including islet 1 . Because the value of $\lambda$ depends on which islet is being used to compare with the others, there is a small difference in the synchronization indices calculated between two islets, such as islet 1 to islet 2 and islet 2 to islet 1 , but these were included in all calculations. Proceeding in this way, $\sim 400$ index values, $\lambda_{1} \ldots \lambda_{400}$, were calculated for a single experimental replicate. Computer code for the calculation of $\lambda$ is available as freeware from www.math.fsu.edu/ $\sim$ bertram/software/islet.

A two-tailed Mann-Whitney test was performed to test for statistical differences of $\lambda$ between the groups of islets exposed to constant and oscillatory glucose stimulations. Unless stated otherwise, all $\lambda$-values are shown as the mean $\pm 1 \mathrm{SD}$.

\section{RESULTS AND DISCUSSION}

Synchronization of the numerous islets within the pancreas is essential for oscillatory insulin output from the pancreas and for efficient glucose utilization by peripheral tissues $(2,6,10$, 15). The mechanism of interislet synchronization is unknown, but a feedback mechanism between the liver and pancreas has been proposed due to similar periods of in vivo glucose and insulin oscillations $(3,16,24)$. In this hypothesis, the glucose levels would become oscillatory as a result of classical insulin/ glucose feedback mechanisms. Because glucose acts as a global signal to all islets within the pancreas, islets become entrained to the oscillatory glucose levels, resulting in synchronized insulin oscillations. We (27) and others $(3,9,18,24)$ have demonstrated entrainment of islet responses to oscillatory glucose levels. However, there is a lack of quantitative evidence of groups of islets synchronizing based on this mechanism. In this report, we demonstrate that glucose oscillations can synchronize multiple islets together as measured by their $\left[\mathrm{Ca}^{2+}\right]_{\mathrm{i}}$.

Microfluidic system. The layout of the microfluidic device utilized in this report to generate the glucose profiles is shown in Fig. 1. As described elsewhere $(27,28)$, two pneumatic pumps were used to create temporal glucose oscillations in the mixing channel, which were delivered to an 860-nl chamber that held $\sim 20$ islets. The benefits of using this device for testing islet synchronization are that it is highly automated, allows complex waveforms to be generated, and allows facile imaging of $\left[\mathrm{Ca}^{2+}\right]_{\mathrm{i}}$.

To ensure that all islets in the chamber would encounter the same glucose concentration simultaneously no matter their location in the 1-mm chamber, the flow from the mixing channel was split and delivered to four inlets to the islet chamber. The inset of Fig. 1 shows an image of 21 islets at the 


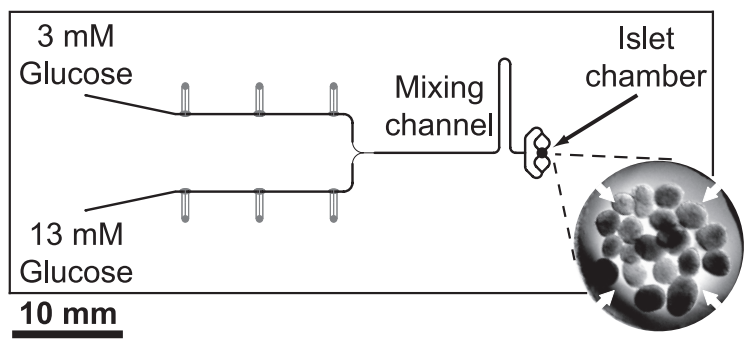

Fig. 1. Layout of the microfluidic perfusion device. Gray lines represent the pneumatic valves used to produce and deliver glucose waveforms to the 1-mm-diameter islet chamber. Glucose waveforms were produced by varying the ratio of 3 and $13 \mathrm{mM}$ glucose delivered from the pumps to the mixing channel. Twenty-one islets are shown in the islet chamber where fura 2 fluorescence from all islets was imaged. The white arrows indicate the direction of flow into the islet chamber.

bottom of the 1-mm-diameter cell chamber, where the white arrows indicate the four locations in which glucose entered the chamber. The time required for the glucose solution to enter the chamber and reach the center was $22 \mathrm{~s}$, as determined by flowing fluorescein through the design and imaging the chamber. Since this time was a small percentage $(7.3 \%)$ of the period of the glucose waves that were to be used in the later experiments, the device was deemed sufficient to test islet synchronization.

Islets are synchronized by glucose waves. In four experiments, the glucose concentration was increased from 3 to 11 $\mathrm{mM}$ and held constant for $\sim 20 \mathrm{~min}$. A representative experiment is shown in Fig. $2 A$, where the glucose concentration (dashed line) is shown on the right $y$-axis and the $\mathrm{F}_{340} / \mathrm{F}_{380}$ signals from all islets are shown as solid lines in different shades of gray and correspond to the left $y$-axis. After the initial rise in $\mathrm{F}_{340} / \mathrm{F}_{380}$ from all islets, the individual islets began to oscillate at different frequencies and became out of phase.
The average $F_{340} / F_{380}$ from all islets in Fig. $2 A$ is shown in Fig. $2 B$. After the initial $\mathrm{F}_{340} / \mathrm{F}_{380}$ response, the average $\mathrm{F}_{340} / \mathrm{F}_{380}$ signal ceased to oscillate due to the unsynchronized islet activity during perfusion with a constant glucose concentration.

After an $\sim 20$-min perfusion with constant $11 \mathrm{mM}$ glucose, a sinusoidal waveform in the glucose concentration was applied, with a median value of $11 \mathrm{mM}$, amplitude of 1 $\mathrm{mM}$, and a period of $5 \mathrm{~min}$ (Fig. 2, $A$ and $B$ ). Within approximately two periods after the application of the sinusoidal glucose waveform, all islets became synchronized by the glucose wave, producing robust oscillations of the average $F_{340} / F_{380}$ ratio that had the same period as the glucose waveform (Fig. 2B). This lag time for islet synchronization was similar to what we observed for individual islets becoming entrained to a glucose waveform (27). These results are representative of four trials using $21,23,22$, and 20 islets that were obtained from one, three, one, and three mice, respectively. We initially hypothesized that it would be easier to synchronize groups of islets from a single mouse, since it has been reported that islets from a single mouse had similar oscillation frequencies $(12,13)$. However, we found that islets from one or three mice were equally susceptible to synchronization.

In two other trials each containing 20 islets from three mice, the opposite glucose profile was applied where the glucose concentration was oscillated immediately after being changed from 3 to $11 \mathrm{mM}$ (Fig. 2, $C$ and $D$ ). After $\sim 20 \mathrm{~min}$ of oscillatory glucose, the islets were perfused with a constant 11 $\mathrm{mM}$ glucose. These experiments also produced strong oscillations of the average $F_{340} / F_{380}$ ratio during the sinusoidal glucose phase and weak and irregular oscillations during the constant glucose phase, indicating that the islets lost synchronization after the glucose waveforms were stopped.

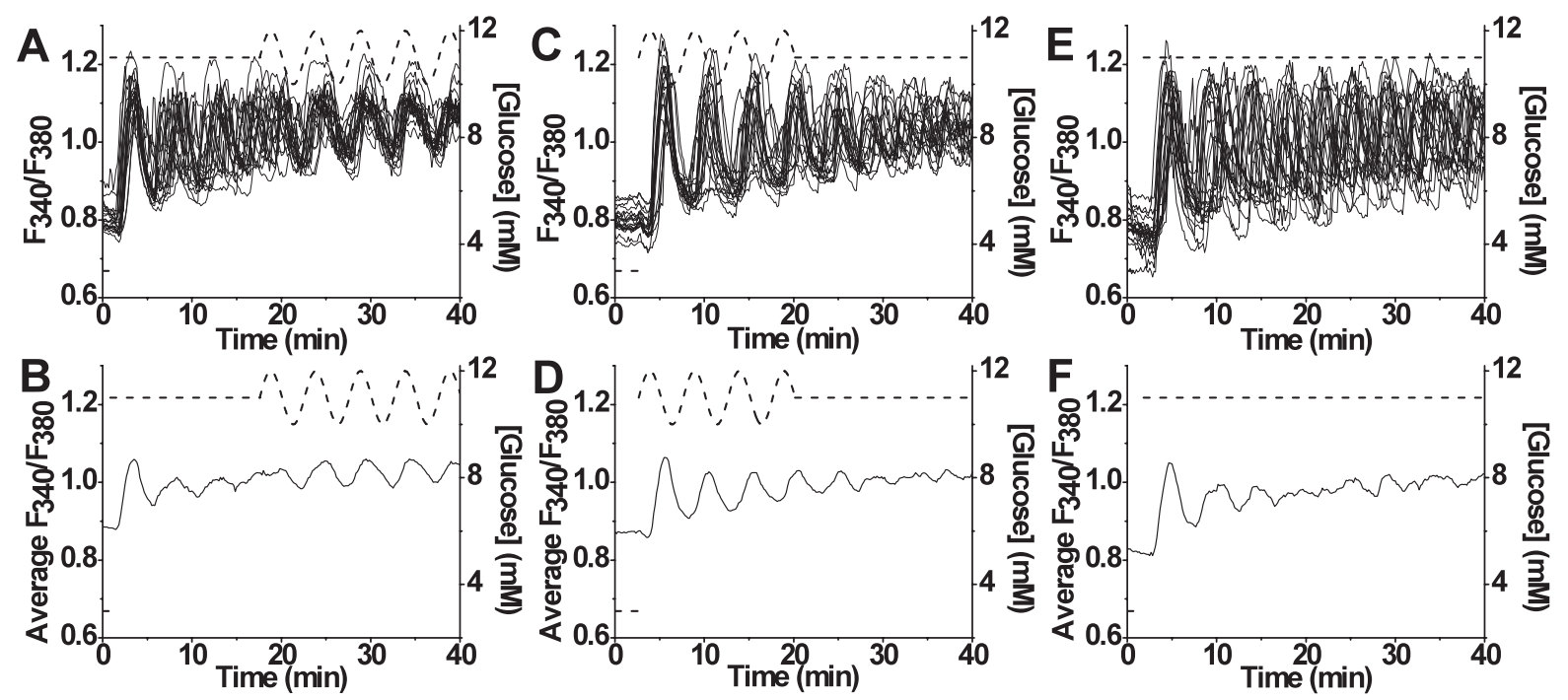

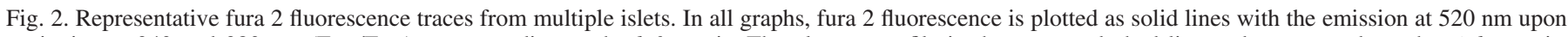

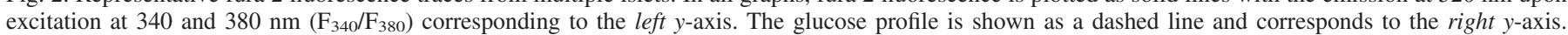

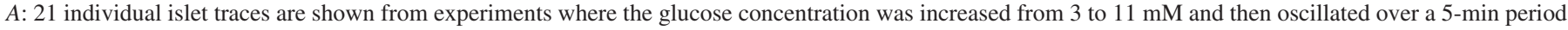

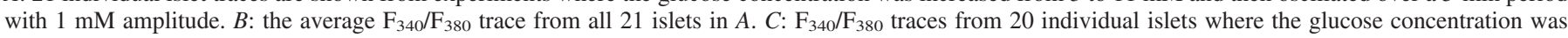

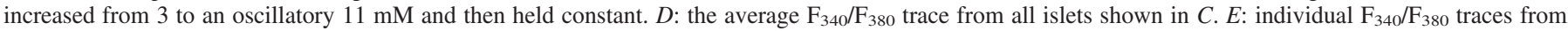

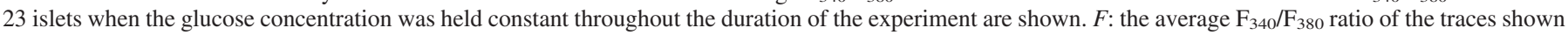
in $E$. 

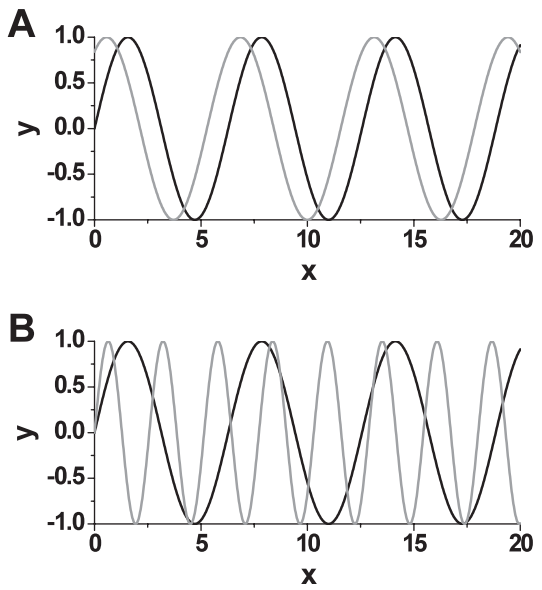

Fig. 3. Illustrative examples for interpretation of the synchronization index. All black traces correspond to $y=\sin (x)$. A: phase-locked oscillations have a high synchronization index; $\lambda=1.00$. Gray trace: $y=\sin (x+1)$. $B$ : the synchronization index is low $(\lambda=0.04)$ when the oscillations phase drift. Gray trace: $y=\sin (2.44 x)$.

Islets are not synchronized when the glucose concentration is constant. To ensure that islets would not have synchronized given more time during application of a constant glucose concentration, four experiments were performed with 31 (2 mice), 21 (1 mouse), 11 (1 mouse), and 23 islets (1 mouse). As shown in Fig. 2, $E$ and $F$, after the glucose concentration was increased from 3 to $11 \mathrm{mM}$, all islets had a large increase and oscillations of the $\mathrm{F}_{340} / \mathrm{F}_{380}$ ratio. However, after $\sim 15 \mathrm{~min}$, the different oscillation frequencies resulted in an average $\mathrm{F}_{340} /$ $\mathrm{F}_{380}$ ratio that was elevated but nonoscillatory, indicating that the islets were unsynchronized.

Analysis of synchronization by spectral analysis and the synchronization index. The synchronization index (20) has been used to examine synchronization of $\beta$-cell $\left[\mathrm{Ca}^{2+}\right]_{\mathrm{i}}$ oscillations $(25,26)$ and was used here as one method for quanti-
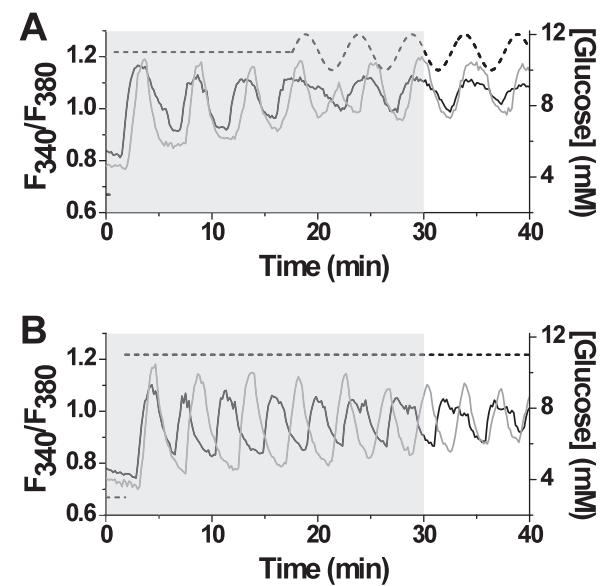

Fig. 4. Examples showing various degrees of synchronization. The synchronization index was calculated over the last $10 \mathrm{~min}$ of the experiment, shown as the unshaded regions in both $A$ and $B$. A : the $\mathrm{F}_{340} / \mathrm{F}_{380}$ traces (black and gray solid lines) from 2 islets taken from Fig. $2 A$ started with a phase shift and then became mostly in phase after the glucose waveform (dashed line) was started, producing a high synchronization index $(\lambda=0.96)$ over the last $10 \mathrm{~min} . B: 2$ $\mathrm{F}_{340} / \mathrm{F}_{380}$ traces taken from 2 islets in Fig. $2 E$ that had little synchrony during perfusion with a constant glucose concentration produced a low synchronization index $(\lambda=0.33)$.
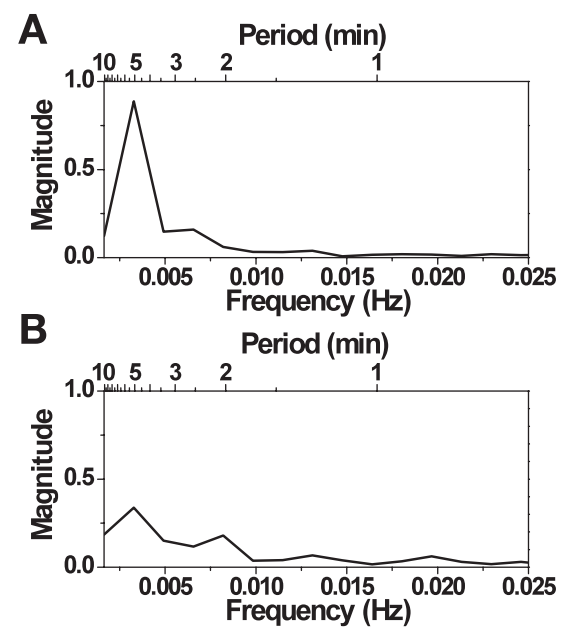

Fig. 5. Spectral analysis of average $\mathrm{F}_{340} / \mathrm{F}_{380}$ traces. $A$ : spectral analysis of the last $10 \mathrm{~min}$ of the data shown in Fig. $2 B$ had a dominant peak at $0.0033 \mathrm{~Hz}$ (bottom $x$-axis) in the fast Fourier transform (FFT) corresponding to a period of 5 min (top $x$-axis). $B$ : The FFT from the last 10 min of data in Fig. $2 F$ when islets were perfused with a constant glucose concentration.

fying the degree of synchrony between $\left[\mathrm{Ca}^{2+}\right]_{\mathrm{i}}$ oscillations in the $\sim 20$ islets measured in each experiment. This index ranges from 0 (not synchronized) to 1 (complete synchronization) and measures the differences in phase between two oscillators over time. Two oscillations with different phases, but with constant phase differences, are completely phase locked or synchronized and receive $\lambda=1$. Two oscillations in which the phase difference changes over time, known as phase drift, receive a value of $\lambda=0$. This measurement of synchrony is independent of the amplitude of the two oscillators.

Illustrative examples of $\lambda$ are shown in Fig. 3, using simple sinusoidal oscillators. In Fig. $3 A$, two sinusoidal oscillators are phase locked, but with a nonzero phase difference. These are considered synchronized, and $\lambda=1.00$. Figure $3 B$ shows two sinusoidal oscillators in which the phase difference changes continuously over time. This example of phase drifting results in a much lower synchronization index, and $\lambda=0.04$. The greater the extent of the phase drift, the lower the value of $\lambda$.

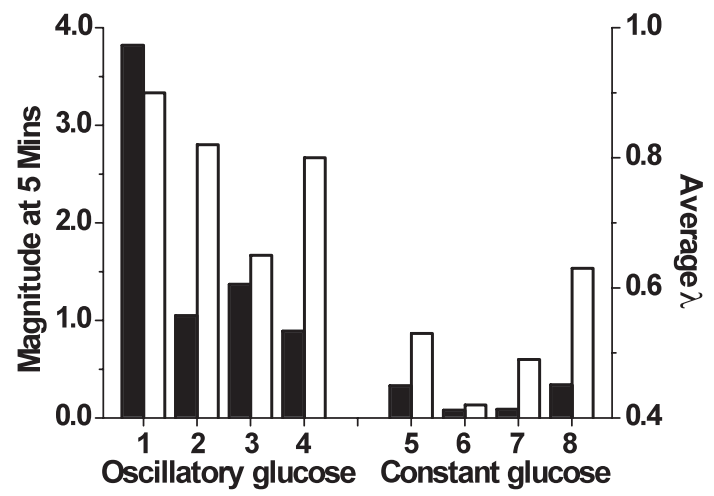

Fig. 6. Summary of the FFT and synchronization index analysis. The filled bars indicate the magnitude of the peak at $0.0033 \mathrm{~Hz}$ (corresponding to a 5-min period) in the FFT of all experiments (left $y$-axis). The open bars indicate the average $\lambda$, shown on the right $y$-axis. There were 4 experiments corresponding to perfusion with an oscillatory glucose concentration (experiments 1-4) and 4 experiments corresponding to perfusion with a constant glucose concentration (experiments 5-8). 
Because the synchronization index was developed to compare two oscillators, we adapted this measurement to our system with $\sim 20$ islet oscillators per experiment by calculating $\lambda$ for each pair of islets (see MATERIALS AND METHODS). This average synchronization index will lie between 0 and 1 and will not likely be at either extreme.

Figure $4 A$ shows $\mathrm{F}_{340} / \mathrm{F}_{380}$ traces from two islets taken from Fig. $2 A$ that were well synchronized during the oscillatory glucose wave. The synchronization index computed with the last $10 \mathrm{~min}$ of data yielded a large value, $\lambda=0.96$, as expected for these highly synchronized oscillators. Figure $4 B$ shows $\mathrm{F}_{340} / \mathrm{F}_{380}$ traces from two islets taken from Fig. $2 E$, when a constant glucose concentration was applied throughout the duration of the experiment. These two islets never synchronized, and $\lambda=0.33$.

To further analyze the fura 2 traces, a spectral analysis was performed on the population mean of the $\mathrm{F}_{340} / \mathrm{F}_{380}$ ratios during the experiments described above to extract the average periods of the oscillations. The FFTs from the data in Fig. 2, $B$ and $F$, are shown in Fig. 5, $A$ and $B$, respectively, as representative examples. In all four experiments where the glucose level was oscillated, the FFT showed a single dominant peak at $0.0033 \mathrm{~Hz}$, corresponding to a period of $5 \mathrm{~min}$ (Fig. 5A), in agreement with the 5-min glucose waveform and the strong oscillations observed during these experiments. In contrast to this result, the FFTs from the four experiments where a constant glucose concentration perfused the islets resulted in low magnitudes at all frequencies in the FFT spectrum due to the weak and irregular average $\mathrm{F}_{340} / \mathrm{F}_{380}$ oscillations (Fig. $5 B$ ).

The magnitude of the FFT trace at $0.0033 \mathrm{~Hz}$, corresponding to a period of $5 \mathrm{~min}$, is shown in Fig. 6 (filled bars) for all experiments. The magnitude of this peak was higher in all experiments where islets were perfused with an oscillatory glucose concentration than the magnitude for perfusion with a constant glucose concentration. The average synchronization indices for these experiments are shown in Fig. 6 (open bars); $\lambda$ was greater for all experiments when oscillatory glucose levels were used compared with perfusion with constant levels and in one case was as large as 0.90 with oscillatory glucose. The synchronization index was $0.78 \pm 0.15$ for islets exposed to oscillatory glucose and $0.52 \pm 0.09$ in constant glucose, which was a significant increase $(P<0.001)$. Together with the spectral analysis, the synchronization index analysis provides strong evidence that the islets were synchronized during exposure to oscillatory glucose concentrations and unsynchronized during exposure to a constant glucose concentration.

In previous work, high-frequency insulin oscillations in vivo $(9,18)$, from perfused pancreas $(3,24)$, and from individual islets or groups of islets $(3,24)$ were entrained to glucose oscillations. The entrainment of these systems implies that the large numbers of islets were synchronized, although the individual islet responses were not measured, as is shown here (Fig. 2). As described in these previous reports and here, the amplitude of the glucose oscillations does not need to be large (5-10\% of the mean), and a range of glucose periods from 5 to $40 \min (5,13,23,29)$ can be used to entrain islet oscillations. The glucose oscillations produced here and in these other reports were meant to mimic in vivo glucose oscillations. How these in vivo glucose oscillations are formed is still unclear, but a model of a pancreas-liver feedback loop has shown that classical insulin-glucose feedback mechanisms can re- sult in glucose oscillations and concomitant synchronization of islets (16).

Conclusion. Synchronization of multiple islets via entrainment to a glucose waveform was demonstrated. Approximately 20 islets from multiple mice were synchronized to an oscillatory glucose waveform at a period similar to what is observed in vivo. Spectral analysis and the synchronization index were used to confirm a degree of synchronization of islets under an oscillatory glucose wave that was greater than a constant glucose concentration.

Our results confirm that glucose waveforms, like those hypothesized to be produced by an in vivo pancreas-liver feedback loop, could synchronize multiple islets. Although this type of feedback loop has been postulated to coordinate ultradian oscillations $(22,23)$, more work will need to be performed to determine whether this type of feedback loop could also generate the type of glucose waveforms described here. In the system described here, paracrine interactions between islets would be present to the same extent in both the oscillatory and constant glucose experiments. Because the islets under constant glucose stimulation did not synchronize, paracrine effects do not seem to have a role in this setting. Future work may utilize islets from diabetic models to investigate how the synchronization of islets from these animals would differ.

\section{ACKNOWLEDGMENTS}

We thank Prof. Wei Wu in the Department of Statistics at Florida State University for helpful discussions on statistical tests and Feng Xian for helpful discussions on the spectral analysis.

\section{GRANTS}

M. G. Roper acknowledges support from the National Institute of Diabetes and Digestive and Kidney Diseases (R01-DK-080714). R. Bertram acknowledges support from the National Institute on Deafness and Other Communication Disorders (R01-DC-002035) and the National Science Foundation (DMS0917664).

\section{DISCLOSURES}

No conflicts of interest, financial or otherwise, are declared by the authors.

\section{REFERENCES}

1. Bertram R, Sherman A, Satin LS. Metabolic and electrical oscillations: partners in controlling pulsatile insulin secretion. Am J Physiol Endocrinol Metab 293: E890-E900, 2007.

2. Bratusch-Marrain PR, Komjati M, Waldhausl WK. Efficacy of pulsatile versus continuous insulin administration on hepatic glucose production and glucose utilization in type I diabetic humans. Diabetes 35: 922-926, 1986.

3. Chou HF, Ipp E. Pulsatile insulin-secretion in isolated rat islets. Diabetes 39: 112-117, 1990.

4. Fendler B, Zhang M, Satin L, Bertram R. Synchronization of pancreatic islet oscillations by intrapancreatic ganglia: a modeling study. Biophys $J$ 97: 722-729, 2009.

5. Gilon P, Shepherd RM, Henquin JC. Oscillations of secretion driven by oscillations of cytoplasmic $\mathrm{Ca}^{2+}$ as evidenced in single pancreatic islets. J Biol Chem 268: 22265-22268, 1993.

6. Komjati M, Bratusch-Marrain P, Waldhausl W. Superior efficacy of pulsatile versus continuous hormone exposure on hepatic glucose production in vitro. Endocrinology 118: 312-319, 1986.

7. Lang DA, Matthews DR, Burnett M, Turner RC. Brief, irregular oscillations of basal plasma insulin and glucose concentrations in diabetic man. Diabetes 30: 435-439, 1981.

8. Longo EA, Tornheim K, Deeney JT, Varnum BA, Tillotson D, Prentki M, Corkey BE. Oscillations in cytosolic free $\mathrm{Ca}^{2+}$, oxygen consumption, and insulin secretion in glucose-stimulated rat pancreatic islets. J Biol Chem 266: 9314-9319, 1991. 
9. Mao CS, Berman N, Roberts K, Ipp E. Glucose entrainment of highfrequency plasma insulin oscillations in control and type 2 diabetic subjects. Diabetes 48: 714-721, 1999.

10. Matthews DR, Naylor BA, Jones RG, Ward GM, Turner RC. Pulsatile insulin has greater hypoglycemic effect than continuous delivery. Diabetes 32: 617-621, 1983.

11. Newsholme P, Gaudel C, McClenaghan NH. Nutrient regulation of insulin secretion and $\beta$-cell functional integrity. In: The Islets of Langerhans, edited by Islam MS. Dordrecht, The Netherlands: Springer, 2010, p. 91-114.

12. Nunemaker CS, Zhang M, Wasserman DH, McGuinness OP, Powers AC, Bertram R, Sherman A, Satin LS. Individual mice can be distinguished by the period of their islet calcium oscillations: is there an intrinsic islet period that is imprinted in vivo? Diabetes 54: 3517-3522, 2005.

13. Nunemaker CS, Dishinger JF, Dula SB, Wu R, Merrins MJ, Reid KR, Sherman A, Kennedy RT, Satin LS. Glucose metabolism, islet architecture, and genetic homogeneity in imprinting of $\left[\mathrm{Ca}^{2+}\right](\mathrm{i})$ and insulin rhythms in mouse islets. PLoS One 4: e8428, 2009.

14. O'Rahilly S, Turner RC, Matthews DR. Impaired pulsatile secretion of insulin in relatives of patients with non-insulin-dependent diabetes. $N$ Engl J Med 318: 1225-1230, 1988.

15. Paolisso G, Scheen AJ, Giugliano D, Sgambato S, Albert A, Varricchio M, D'Onofrio F, Lefèbvre PJ. Pulsatile insulin delivery has greater metabolic effects than continuous hormone administration in man: importance of pulse frequency. J Clin Endocrinol Metab 72: 607-615, 1991.

16. Pedersen MG, Bertram R, Sherman A. Intra- and inter-islet synchronization of metabolically driven insulin secretion. Biophys $J$ 89: 107-119, 2005.

17. Polonsky KS, Given BD, Hirsch LJ, Tillil H, Shapiro ET, Beebe C, Frank BH, Galloway JA, Van Cauter E. Abnormal patterns of insulinsecretion in non-insulin-dependent diabetes-mellitus. New Engl J Med 318: 1231-1239, 1988.

18. Pørksen N, Juhl C, Hollingdal M, Pincus SM, Sturis J, Veldhuis JD, Schmitz O. Concordant induction of rapid in vivo pulsatile insulin secretion by recurrent punctuated glucose infusions. Am J Physiol Endocrinol Metab 278: E162-E170, 2000.

19. Ritzel RA, Veldhuis JD, Butler PC. The mass, but not the frequency, of insulin secretory bursts in isolated human islets is entrained by oscillatory glucose exposure. Am J Physiol Endocrinol Metab 290: E750-E756, 2006.

20. Rosenblum R, Pikovsky A, Kurths J, Schäfer C, Tass PA. Phase synchronization: from theory to data analysis. In: Neuro-Informatics and Neural Modelling, edited by Moss F and Gielen S. Amsterdam: Elsevier, 2001, p. 279-322.

21. Stagner JI, Samols E. Role of intrapancreatic ganglia in regulation of periodic insular secretions. Am J Physiol Endocrinol Metab 248: E522E530, 1985.

22. Sturis J, Van Cauter E, Blackman JD, Polonsky KS. Entrainment of pulsatile insulin secretion by oscillatory glucose infusion. J Clin Invest 87 : 439-445, 1991.

23. Sturis J, Polonsky KS, Mosekilde E, Van Cauter E. Computer model for mechanisms underlying ultradian oscillations of insulin and glucose. Am J Physiol Endocrinol Metab 260: E801-E809, 1991.

24. Sturis J, Pugh WL, Tang JP, Ostrega DM, Polonsky JS, Polonsky KS. Alterations in pulsatile insulin secretion in the Zucker diabetic fatty rat. Am J Physiol Endocrinol Metab 267: E250-E259, 1994.

25. Zarkovic M, Henquin JC. Synchronization and entrainment of cytoplasmic $\mathrm{Ca}^{2+}$ oscillations in cell clusters prepared from single or multiple mouse pancreatic islets. Am J Physiol Endocrinol Metab 287: E340-E347, 2004.

26. Zhang M, Fendler B, Peercy B, Goel P, Bertram R, Sherman A, Satin L. Long lasting synchronization of calcium oscillations by cholinergic stimulation in isolated pancreatic islets. Biophys $J$ 95: 4676-4688, 2008.

27. Zhang X, Grimley A, Bertram R, Roper MG. Microfluidic system for generation of sinusoidal glucose waveforms for entrainment of islets of Langerhans. Anal Chem 82: 6704-6711, 2010.

28. Zhang X, Roper MG. Microfluidic perfusion system for automated delivery of temporal gradients to islets of Langerhans. Anal Chem 81: 1162-1168, 2009. 